J. Tant and J. Driesen, “Accurate second-order interpolation for power electronic circuit simulation," Proc. IEEE $18^{\text {th }}$ Workshop on Control and Modeling for Power Electronics (COMPEL) 2017, Stanford, CA, USA, Jul. 9-12, 2017, 8 pages.

Digital Object Identifier: 10.1109/COMPEL.2017.8013336

URL: http://ieeexplore.ieee.org/document/8013336/

(C) 2017 IEEE. Personal use of this material is permitted. Permission from IEEE must be obtained for all other users, including reprinting/ republishing this material for advertising or promotional purposes, creating new collective works for resale or redistribution to servers or lists, or reuse of any copyrighted components of this work in other works. 


\title{
Accurate Second-Order Interpolation for Power Electronic Circuit Simulation
}

\author{
Jeroen Tant and Johan Driesen \\ Department of Electrical Engineering \\ KU Leuven - Energyville \\ Leuven, Belgium \\ Email: jeroen.tant@esat.kuleuven.be
}

\begin{abstract}
This paper presents a second-order accurate method for circuit simulation with idealized power electronic switches. The method combines the integration method TR-BDF2 with an improved quadratic interpolation technique for the localization of switch events. Next to preserving second-order accuracy after interpolation, the technique also preserves the capability to damp fast transients caused by small on-resistances and large offresistances. Conventional interpolation techniques for integration methods do not have this damping property. This results in a loss of accuracy if a switch event occurs shortly after a transition to discontinuous conduction mode. Consequently, the step size required to achieve the desired level of accuracy is sometimes smaller than expected. With the improved interpolation technique, the second-order accuracy associated with TR-BDF2 is not affected at switch events. Numerical experiments confirm the improved accuracy of the proposed method. The method is compared with the simulation tools PSIM and PSCAD, and with the conventional interpolation polynomial of TR-BDF2 as used in Simulink.
\end{abstract}

\section{INTRODUCTION}

Power electronic circuit simulation tools often use idealized switch models instead of detailed semiconductor device models to speed up the simulation [1]-[4]. Simulation studies at this level of detail are used to verify the operation of a converter circuit, to design the control system, to assess the quality of waveform conversion, and to assess the impact on the surrounding electrical system. In the field of power systems, simulation tools for electromagnetic transient simulation also support idealized switches to accommodate the analysis of power electronic equipment in the transmission or distribution grid [5]-[9]. Although electromagnetic transient simulation is usually covered separately in literature within the field of power systems, these tools are in essence similar to the circuit simulation tools for power electronics. The main differences are in the terminology, the scope of applications, and the provided library of components.

Circuit simulation tools supporting idealized switches require specialized numerical solution techniques [3], [4], [6], [9]-[12]. Idealized switches are either completely ideal switches, for which both the on-resistance and off-conductance are zero, or almost ideal switches, for which the on-resistance and off-conductance is small. Several difficulties arise with completely ideal switches because the circuit topology changes after switch events [4], [10], [11]. Such difficulties include changes in the number of effective state variables and the occurrence of impulsive and underdetermined switch configurations at intermediate stages in the solution algorithm. Methods that cope with these difficulties by using specialized algorithms are further referred to as following the variable structure approach. Example tools with a variable structure approach are PLECS [4], [13] and SimPowerSystems if the option "Enable use of ideal switching devices" is enabled [14]. An advantage of the variable structure approach is that stiff models can sometimes be avoided through careful modeling, so that explicit integration methods remain efficient. The variable structure approach is not within the scope of this paper, but the proposed method may also be beneficial for this approach if the model is stiff.

The alternative approach, referred to as the constant structure approach, enforces a constant circuit topology by using small on-resistances and large off-resistances. The main advantage of this approach is that there is no overhead associated with algorithms to resolve topology changes at switch events. A disadvantage is that resulting models are often stiff. When an inductor current is blocked by a diode, for instance, the large off-resistance often causes switching transients in some circuit variables which are damped so fast that they appear as instantaneous jumps at the beginning of a step. To avoid excessively small step sizes, an integration method is required capable of damping out these transients almost instantly in one step. $L$-stable integration methods such as the first-order backward Euler method have this capability, but the secondorder trapezoidal method does not [15]. As a result, the trapezoidal method often exhibits slowly decaying numerical oscillations [1], [8]. Some tools, including EMTP-RV (with the "Trapezoidal and Backward Euler" integration option) and PSIM, therefore perform one or two backward Euler steps after switch events to suppress numerical oscillations [3], [6], [16]-[18]. But they do this at the cost of losing the secondorder accuracy of the trapezoidal method. Other tools use a second-order $L$-stable method. XTAP, for instance, uses the method 2s-DIRK [19], while the Simulink solver ode23tb, an implementation of TR-BDF2 [20], is recommended in the manual of SimPowerSystems [14] and also available in PLECS [13] for stiff problems.

Switch events do not generally occur at the beginning or end of a time step. To avoid inaccuracies, a common approach is to interrupt the simulation and locate the event within a 
completed step through interpolation [1], [6], [9], [12], [21][23]. The simulation is then rolled back and restarted from this event location so that the beginning of the following step is synchronized with the associated switch action. The tool PSCAD, for instance, uses linear interpolation [6], [22]. With linear interpolation, the second-order accuracy of the trapezoidal method is lost again. Interpolation is also used in Simulink for zero crossing detection, which is employed by both SimPowerSystems and PLECS to locate switch events [13], [14]. The variable-step integration methods of Simulink come with an embedded interpolation polynomial which preserves the integration order [24].

This paper focuses on the consequences of using interpolation on an $L$-stable integration method. It will be shown in Section IV that the beneficial property of $L$-stability is generally not preserved with interpolation. As a result, most methods lose their capability to damp fast switching transients instantaneously in steps with interpolation. The examples of this paper illustrate that this causes inaccuracies in particular cases. Therefore, a new quadratic interpolation method for TRBDF2 is proposed in Section V, which preserves second-order accuracy and the capability to damp fast switching transients. In Section VI, the accuracy of the method is compared with the regular interpolation polynomial for TR-BDF2 and the existing tools PSCAD and PSIM. Sections II and III first introduce the circuit model and numerical simulation with the constant structure approach.

\section{Circuit Model with Idealized Switches}

\section{A. DAE Representation}

The circuit and control system equations are considered as a system of differential algebraic equations (DAEs) of form

$$
\begin{aligned}
\dot{\theta} & =1, \\
\dot{x} & =f(x, y, \theta, m, s), \\
0 & =g(x, y, \theta, m, s),
\end{aligned}
$$

where the elements of $x, y, \theta, m$, and $s$ are the dynamic (differentiated) variables, the algebraic (non-differentiated) variables, the timer states, the discrete-time memory states, and the binary switch configuration states respectively.

The circuit equations are obtained with a formulation technique such as sparse tableau analysis, nodal analysis, modified augmented nodal analysis, or branch analysis. Some algebraic variables are labeled as positive guard variables for which an event is triggered when they cross zero. At such events, it is possible to reinitialize the value of timer states, memory states and switch states. Because the definition of such reinitializations can be considered externally from the continuoustime simulation, they are not included in the model. For components with piecewise-defined characteristics such as ideal diodes, associated switch states are simply toggled if their corresponding guard variable crosses zero.

\section{B. DAE Structure for a Fixed Switch Configuration}

Between events, $m$ and $s$ are constant. In literature, DAEs for which (1c) uniquely determines $y$ as an implicit function of $x$ and $\theta$ for fixed $m$ and $s$ are said to have index one [15], [25]-[27]. Then, (1b) can be treated as an ordinary differential equation (ODE) for which each evaluation of $f$ involves solving (1c) as a system of equations. As a result, integration methods for ODEs are directly applicable.

Unfortunately, (1c) is not uniquely solvable on its own for many circuits of practical interest. This happens, for instance, when two capacitors are connected in parallel, when two inductors are connected in series, or when a capacitor is connected in parallel with a voltage source. In this case, some initial values for the dynamic variables cannot be freely chosen because $g$ imposes a constraint on $x$. Nevertheless, wellposed DAEs are always equivalent to an underlying index-one problem, which can often be revealed using index reduction techniques [15], [26].

In the following, index reduction is explained for the case where $f$ and $g$ are linear in $x$ and $y$. The equations are formulated as

$$
\begin{aligned}
& \dot{x}=A_{s} x+B_{s} y+F u(\theta, m, s), \\
& 0=C_{s} x+D_{s} y+G u(\theta, m, s),
\end{aligned}
$$

where $u$ is a vector-valued input function that is smooth in $\theta$. The discussed algorithm is based on [28]. Similar algorithms are found in $[29$, p. 154] and [26, p. 20]. The algorithm is executed for a given switch state $s$. In general, the outcome of the algorithm may depend on $s$. If $D_{s}$ is invertible, the DAE is already of index one and the algorithm ends. Otherwise, it is possible to obtain zero rows in the matrix $D$ using elementary row operations, resulting in

$$
\begin{aligned}
& \dot{x}=B_{s} y+A_{s} x+F u(\theta, m, s), \\
& 0=D_{1, s} y+C_{1, s} x+G_{1} u(\theta, m, s), \quad+\tilde{C}_{2, s} x+\tilde{G}_{2} u(\theta, m, s), \\
& 0=\quad
\end{aligned}
$$

so that (3c) reveals a hidden constraint involving only dynamic variables and known input variables. The algorithm then selects dependent dynamic variables and relabels them as algebraic variables. Afterwards, the corresponding equations in (3a) are replaced with the derivative of (3c), which is

$$
0=\tilde{C}_{2, s}\left(A_{s} x+B_{s} y+F u(\theta, m, s)\right)+\tilde{G}_{2} \dot{u}(\theta, m, s) .
$$

The resulting problem is again of form (2). If the new $D_{s}$ is invertible, the reduced problem has index one, the original problem is said to have index two, and the algorithm ends. Otherwise, the procedure must be repeated.

The reduced problem always has fewer dynamic variables than the original problem, because interdependencies on $x$ are removed. The initial conditions for the remaining dynamic variables can be freely chosen. Furthermore, derivatives of the input signals may be required due to the addition of (4) in the system of equations. For instance, if a capacitor is connected in parallel with a time-dependent voltage source, the current through that capacitor will be proportional to the derivative of the voltage. 
After reducing the index to one, the underlying state-space formulation for the given switch configuration becomes

$$
\begin{aligned}
& \dot{x}=\left(A_{s}-B_{s} D_{s}^{-1} C_{s}\right) x+\left(F-B_{s} D_{s}^{-1} G\right) u(\theta, m, s), \\
& y=-D_{s}^{-1} C_{s} x-D_{s}^{-1} G u(\theta, m, s) .
\end{aligned}
$$

The state-space formulation can be solved as a regular ODE with output equations. However, it is not always advisable to compute the state-space matrices explicitly, because the sparsity of the equations is then generally lost.

\section{Variable and Constant Structure}

With completely ideal switches, the DAE structure can vary with the switch configuration. For instance, the current of an inductor can be constrained to zero by a diode or two capacitors can be connected in parallel by a diode. As a consequence, methods based on the variable structure approach must apply the index reduction algorithm for every encountered switch configuration. PLECS, for instance, recomputes a statespace form for every encountered switch configuration using an algorithm similar to the index reduction algorithm of Section II-B [30].

With the constant structure approach, a fixed circuit topology is assumed so that the DAE structure does not change. This can be achieved by assuming small on-resistances and large off-resistances for every switch element. If index reduction is required, the algorithm needs to be executed only once at the beginning of the simulation.

\section{Simulation with the Constant Structure APPROACH}

\section{A. Numerical Integration between Switch Events}

For a given switch configuration, the equations are sufficiently smooth so that numerical integration methods for DAEs are applicable. A numerical integration method is inserted directly in (1), without index reduction, and the resulting sparse system of equations is solved as a whole in each step [15], [26]. Not every integration method is suitable to solve DAEs with index-two or above [15], [26]. Some methods for stiff problems capable of solving index-two DAEs directly are: the trapezoidal method, the backward difference formula (BDF) family of methods (including backward Euler as BDF1), and TR-BDF2 [15], [26]. The trapezoidal method, the method of Gear (BDF2), and TR-BDF2 are often used in circuit simulation [1], [5], [6], [31], [32].

The scope of this paper is limited to methods with a fixed step size. Variable-step implementations are applied in SPICElike simulators for integrated circuits [31], [32]. Methods with a variable step size have the advantage that the step size can be adapted locally to track highly nonlinear dynamics of semiconductor device models.

In power electronic circuit simulation with idealized switches, a piecewise linear approach is followed instead of modeling nonlinear semiconductor dynamics in detail. With a fixed step-size, it is possible to reuse $L U$-factorizations of the sparse system matrix in successive steps, as long as the switch configuration does not change. This approach is also common in electromagnetic transient simulation [6], [7].

\section{B. Initialization and Reinitialization After Switch Events}

The trapezoidal method and TR-BDF2 require initial values for the algebraic variables $y$ in addition to the user-supplied initial values for the dynamic variables $x$. Values for $y$ must also be reinitialized after switch events because they can be discontinuous [12]. Reinitialized values for $y$ are computed by solving (1c) as a system of equations for given values of $x$. For index-two DAEs, index reduction is applied once at the beginning of the simulation.

This system of equations for reinitialization is different form the system resulting from discretizing (1) with the integration method. Therefore, the solver for reinitialization is considered separately for the solver for numerical integration.

Because switch events may trigger other events, reinitialization is applied sequentially until a valid switch configuration is found.

\section{Event Detection and Localization}

If a guard variable becomes negative at the end of a step, an interpolation polynomial of the guard variable is used to determine the moment of zero-crossing within the step. Afterwards, the whole solution is rolled back to the moment zero-crossing through interpolation of $x$ and $y$. After executing the associated switch actions, reinitialization is performed and the simulation continues. Some methods for interpolation are discussed in the next section.

\section{EFFECT OF INTERPOLATION ON THE L-STABILITY PROPERTY OF INTEGRATION METHODS}

\section{A. Backward Euler with Linear Interpolation}

The backward Euler method is a first-order method with a single implicit stage. Applied to (1), with $\theta, s$ and $m$ omitted for clarity, the backward Euler formula becomes

$$
\begin{aligned}
x_{k+1} & =x_{k}+h f\left(x_{k+1}, y_{k+1}\right), \\
0 & =g\left(x_{k+1}, y_{k+1}\right),
\end{aligned}
$$

where $h$ is the fixed step size. The computation of a step involves solving (6) as a system of equations.

Backward Euler applied to the linear scalar test equation $\dot{x}=\lambda x$ with $\lambda \in \mathbb{C}$ gives

$$
x_{k+1}=\Phi(h \lambda) x_{k}, \text { with } \Phi(h \lambda)=\frac{1}{1-h \lambda} .
$$

The function $\Phi(h \lambda)$ is called the stability function associated with this method [15]. The stability region corresponding to a stability function is the set $\{z \in \mathbb{C}|| \Phi(z) \mid \leq 1\}$ in the complex plane [15]. It is the set of values for $h \lambda$ for which the numerical solution of the test equation is stable. The backward Euler method is $A$-stable because its stability region includes the left half-plane [15]. This means that if $\dot{x}=\lambda x$ is stable, the numerical solution will be stable too for any step size. 
Furthermore, backward Euler is also $L$-stable because it has the property

$$
\lim _{h \lambda \rightarrow \infty} \frac{1}{1-h \lambda}=0
$$

in addition to being $A$-stable. As a result of $L$-stability, solution components with a very small time constant relative to the step size $h$ will be damped out almost instantly. This property is favorable if there are fast switching transients in the algebraic variables for which a detailed simulation with smaller steps is not desired.

We now investigate how $L$-stability is affected by linear interpolation at $t_{k}+\alpha h$ with $0 \leq \alpha \leq 1$. The limit of the resulting interpolated stability function as a function of $\alpha$ is given by

$$
\lim _{h \lambda \rightarrow \infty} 1-\alpha+\frac{\alpha}{1-h \lambda}=1-\alpha,
$$

and is only zero for $\alpha=1$. Hence, $L$-stability is not preserved with interpolation except when taking a full step.

\section{B. Trapezoidal Method with Quadratic Interpolation}

The scheme of the second-order trapezoidal method is

$$
\begin{aligned}
x_{k+1} & =x_{k}+\frac{h}{2} f\left(x_{k}, y_{k}\right)+\frac{h}{2} f\left(x_{k+1}, y_{k+1}\right), \\
0 & =g\left(x_{k+1}, y_{k+1}\right) .
\end{aligned}
$$

One implicit stage has to be solved at each step. In contrast with the backward Euler method, both $x_{k}$ and $y_{k}$ are needed to compute $x_{k+1}$ and $y_{k+1}$.

The stability function corresponding to the trapezoidal method is

$$
\Phi(h \lambda)=\frac{1+\frac{h}{2} \lambda}{1-\frac{h}{2} \lambda},
$$

and the associated stability region is exactly equal to the left half-plane. The trapezoidal method is therefore $A$-stable. The method is not $L$-stable because

$$
\lim _{h \lambda \rightarrow \infty} \frac{1+\frac{h}{2} \lambda}{1-\frac{h}{2} \lambda}=-1 .
$$

Because the above limit approaches -1 as $h \lambda$ approaches infinity, a solution component for which $h \lambda$ is too large will undergo a sign reversal in every step. This sign reversal causes numerical oscillations [15].

A quadratic interpolation formula for the trapezoidal method is obtained with Hermite interpolation [33] as

$$
\tilde{x}(\alpha)=\left(1-\alpha^{2}\right) x_{k}+\alpha^{2} x_{k+1}+\left(\alpha-\alpha^{2}\right) h f\left(x_{k}, y_{k}\right)
$$

This interpolation formula preserves second-order accuracy. Hermite interpolation cannot be applied directly to the algebraic variables $y$, because the derivatives are not available. Interpolated values for $y$ can be obtained by solving the reinitialization problem of Section III-B for each required value of $\alpha$.
The behavior at infinity of the stability function after quadratic interpolation is determined by the limit

$$
\lim _{h \lambda \rightarrow \infty} 1-\alpha^{2}+\alpha^{2} \frac{1+\frac{h}{2} \lambda}{1-\frac{h}{2} \lambda}+\alpha(1-\alpha) h \lambda,
$$

which only exists if $\alpha=0$ or $\alpha=1$. Consequently, $A$-stability is not preserved by quadratic interpolation.

The tool PSCAD uses the trapezoidal method with linear interpolation, at the cost of loosing second order accuracy in interpolated steps. The limit behavior at infinity of the linearly interpolated stability function is expressed as

$$
\lim _{h \lambda \rightarrow \infty} 1-\alpha+\alpha \frac{1+\frac{h}{2} \lambda}{1-\frac{h}{2} \lambda}=1-2 \alpha,
$$

which indicates that the method preserves $A$-stability and is $L$-stable only for $\alpha=0$ or $\alpha=1 / 2$. This explains why a trapezoidal step with half-step linear interpolation will successfully suppress numerical oscillations. In the tool PSCAD/EMTDC, for instance, such a half-step interpolation is applied after every switch event [6], [22], [34]. Linear interpolation is directly applicable to both $x$ and $y$.

\section{TR-BDF2 with Quadratic Interpolation}

TR-BDF2 was introduced in [32] for the transient simulation of semiconductor devices. The method is available as a general-purpose integration method in Simulink under the name ode23tb [35]. TR-BDF2 combines an internal trapezoidal step and an internal BDF2 step into a single-step method [20], [32]. The resulting method has order two and inherits the $L$-stability of the BDF2 step. The formula is given by

$$
\begin{aligned}
x_{k+\gamma} & =x_{k}+\gamma \frac{h}{2} f\left(x_{k}, y_{k}\right)+\gamma \frac{h}{2} f\left(x_{k+\gamma}, y_{k+\gamma}\right), \\
0 & =g\left(x_{k+\gamma}, y_{k+\gamma}\right), \\
x_{k+1} & =(1-\eta) x_{k}+\eta x_{k+\gamma}+\gamma \frac{h}{2} f\left(x_{k+1}, y_{k+1}\right), \\
0 & =g\left(x_{k+1}, y_{k+1}\right),
\end{aligned}
$$

where $\gamma=2-\sqrt{2}$ and $\eta=\frac{1+\sqrt{2}}{2}$ [32]. The internal trapezoidal step has size $\gamma h$, the subsequent internal BDF2 step has size $(1-\gamma) h$. Two implicit stages must be solved to compute one step. The factor $\gamma \frac{h}{2}$ in front of $f$ is same for both implicit stages, allowing to reuse the $L U$ factorization of the system matrix for both stages. The associated stability function is

$$
\Phi(h \lambda)=\frac{1-\eta}{1-\frac{\gamma}{2} h \lambda}+\frac{\eta\left(1+\frac{\gamma}{2} h \lambda\right)}{\left(1-\frac{\gamma}{2} h \lambda\right)^{2}}=\frac{1+\sqrt{2} \frac{\gamma}{2} h \lambda}{\left(1-\frac{\gamma}{2} h \lambda\right)^{2}} .
$$

An order-preserving Hermite interpolant for TR-BDF2 is provided in [20], but Hermite interpolation leads to similar problems as with the trapezoidal method. Because the intermediate trapezoidal stage is also second-order accurate, an alternative is to use the quadratic Lagrange interpolant through $x_{k}, x_{k+\gamma}$, and $x_{k+1}$. The resulting interpolation function is

$$
\begin{aligned}
& \tilde{x}(\alpha)=p_{1}(\alpha) x_{k}+p_{2}(\alpha) x_{k+\gamma}+p_{3}(\alpha) x_{k+1}, \\
& \tilde{y}(\alpha)=p_{1}(\alpha) y_{k}+p_{2}(\alpha) y_{k+\gamma}+p_{3}(\alpha) y_{k+1},
\end{aligned}
$$




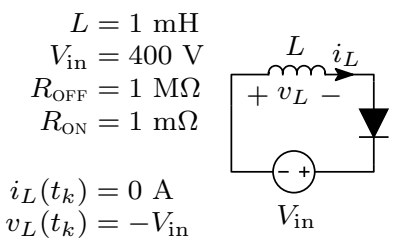

(a)

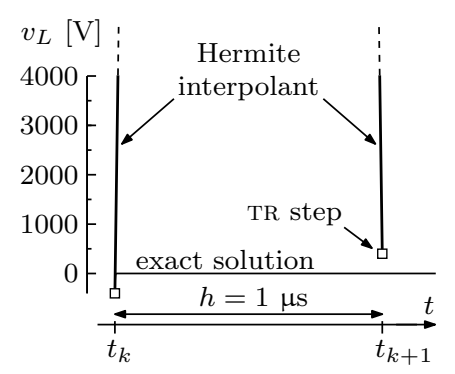

(c)

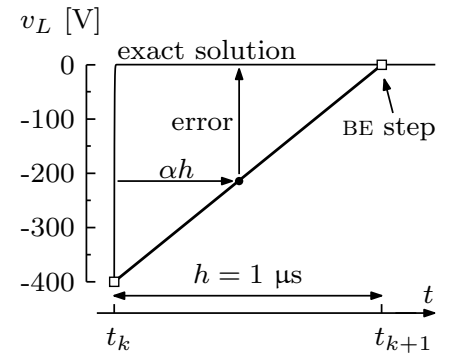

(b)

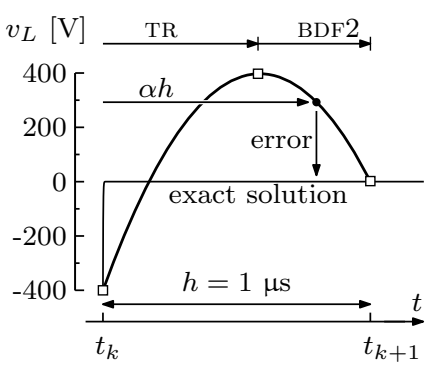

(d)
Fig. 1. Example circuit at diode turn-off (a). The error introduced when interpolation is performed in the subsequent step of size $h=1 \mu \mathrm{s}$, for backward Euler with linear interpolation (b), for the trapezoidal method with quadratic Hermite interpolation (c) and for TR-BDF2 with quadratic Lagrange interpolation (d)).

with

$$
\begin{aligned}
& p_{1}(\alpha)=\gamma^{-1}(\alpha-\gamma)(\alpha-1) \\
& p_{2}(\alpha)=\frac{\alpha(\alpha-1)}{\gamma(\gamma-1)}, \quad \text { and } \quad p_{3}(\alpha)=\frac{\alpha(\alpha-\gamma)}{(1-\gamma)}
\end{aligned}
$$

An important advantage is that the interpolation function is also applicable to the algebraic variables. It can be verified in Simulink, by enabling the optional refinement points in the solution output, that this alternative Lagrange interpolant is also the one used for ode23tb.

The limit behavior at infinity of the interpolated stability function is expressed as

$$
\lim _{h \lambda \rightarrow \infty} p_{1}(\alpha)+p_{2}(\alpha) \frac{1+\frac{\gamma}{2} h \lambda}{1-\frac{\gamma}{2} h \lambda}+p_{3}(\alpha) \frac{1+\sqrt{2} \frac{\gamma}{2} h \lambda}{\left(1-\frac{\gamma}{2} h \lambda\right)^{2}} .
$$

For this stability function, $A$-stability is preserved, but $L$ stability is only achieved if a full step is performed or if $\alpha=2 \gamma^{2}=3-2 \sqrt{2}$.

\section{Consequences of Loosing L-Stability in Interpolated Steps}

To illustrate the consequences in the context of power electronic circuit simulation, consider the example circuit in Fig. 1a. The diode just turned off at $t_{k}$, so that $i_{L}\left(t_{k}\right)=0$ and $v_{L}\left(t_{k}\right)=-400 \mathrm{~V}$. Both $i_{L}$ and $v_{L}$ then undergo a quickly damped transient with time constant $L / R_{\text {off }}$. While $i_{L}$ settles at a current of only $-400 \mu \mathrm{A}$, the algebraic variable $v_{L}$ undergoes a jump from $-400 \mathrm{~V}$ to $0 \mathrm{~V}$. This circuit is simulated for a single step with backward Euler in Fig. 1 b. To avoid the extremely small step size required to track the transient in $v_{L}$ accurately, $h$ is chosen sufficiently large so that the fast transient is damped instantaneously. If linear interpolation is applied afterwards in this step, the result is no longer damped because the $L$-stability property is lost. The trapezoidal method does not damp the fast transient and initiates the first step of a numerical oscillation, as seen in Fig. 1c. The use of the Hermite interpolant in this step results in a large deviation from the exact solution. In Fig. 1d, TRBDF2 succeeds in damping the fast transient in a single step, but interpolation in this step is still not accurate.

In practice, there are particular situations for which this problem leads to inaccurate output or inaccurate event localization. First, if a second switch event occurs shortly after an inductor went into discontinuous conduction mode, the interpolation of the second event might occur in the first step after the first event. This interpolation is then not reliable. Second, if resynchronization with the original sampled time grid is desired, interpolation is required in every first step after a switch event, again resulting in inaccurate interpolation. Finally, if an inductive current through a diode bridge crosses zero, there will be an instantaneous moment of discontinuous conduction where all diodes are blocking before two diodes turn on. The localization of the turn-on event might be delayed due to inaccurate interpolation.

\section{PRoposed Method}

\section{A. TR-BDF2 Stages}

The proposed method uses TR-BDF2 with step size $h$ : internal trapezoidal stages of size $\gamma h$ are alternated with internal BDF2 stages of size $(1-\gamma) h$. If there are no switch events, only the output of BDF2 is considered as a full-step solution point.

If an event occurs in one of the internal stages, interpolation is performed using quadratic Lagrange interpolation with the output of two previous stages. After switch events, a full TR-BDF2-TR sequence is always executed. The output of the first internal trapezoidal step after a switch event is not reliable because fast solution components are not yet damped. Therefore, the interpolation technique as proposed in the next section is used in the first three stages after each switch event.

\section{B. Interpolation Method for TR-BDF2-TR Sequence After Each Switch Event}

To preserve sufficient damping of fast transients after interpolation in the first TR-BDF2-TR sequence after a switch event, for instance at $t=t_{k}$, quadratic Lagrange interpolation is performed through three $L$-stable solution points. The first solution point is the output of the $L$-stable BDF2 stage located at $t_{k+1}=t_{k}+h$. The second solution point is obtained by evaluating the quadratic interpolant (18) on the TR-BDF2 sequence with $\alpha=\alpha_{L}=3-2 \sqrt{2}$, one of the two roots of (19). The third solution point is the output of the final TR-stage in the sequence at $t_{k+1}+\gamma$, which inherits the $L$-stability of the preceding BDF2 stage. Fig. 2 illustrates the method applied to the example of Fig. 1. 


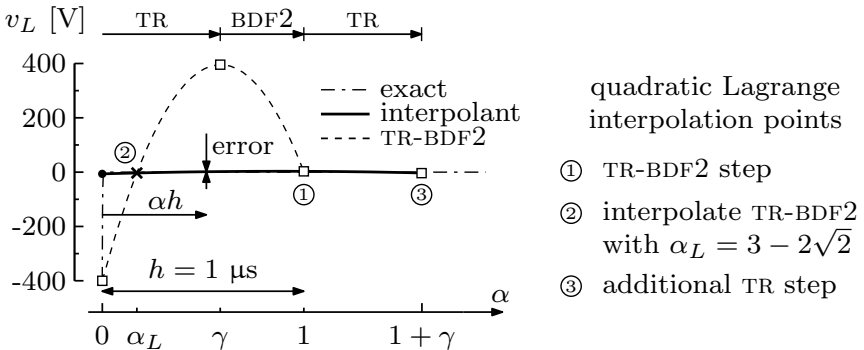

Fig. 2. The proposed quadratic interpolant applied to the example of Fig. 1a.

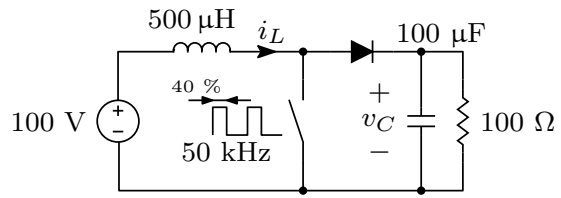

$$
\begin{aligned}
R_{\mathrm{OFF}} & =10 \mathrm{M} \Omega \\
R_{\mathrm{ON}} & =1 \mathrm{~m} \Omega \\
i_{L}(0) & =0 \mathrm{~A} \\
v_{C}(0) & =260 \mathrm{~V}
\end{aligned}
$$

Fig. 3. Boost converter test circuit with a fixed duty cycle.

In general, there will be a discontinuous gap at $t=t_{k}$, because the proposed interpolant does not pass through $x_{k}$ and $y_{k}$. The size of this gap is $O\left(h^{3}\right)$ in asymptotic notation, following from the second-order accuracy of the integration method and quadratic interpolation. Therefore, the size of the gap will decrease when the step size is sufficiently reduced. A large gap indicates that the step size is too large to accurately follow the transient of the respective variable. However, if fast solution components of a variable are strongly damped, and if these solution components are deliberately not simulated in detail, a large gap is exactly what is desired. The discontinuity at $\alpha=0$ is then exploited to approximate the fast solution components as if they jump instantaneously.

Because the discontinuity is interpreted as an instantaneous transition, all values traversed in the gap should also be considered as part of the solution. For instance, it is possible that a switch event is triggered in the instantaneous transition for which interpolation of the variables to the zero-crossing is required.

\section{Numerical COMPARison with Existing Methods}

\section{A. Boost Converter in Discontinuous Conduction Mode}

To assess the effectiveness of the proposed method, the boost converter circuit in Fig. 3 is simulated for $0.4 \mathrm{~ms}$ with PSCAD, PSIM, the regular TR-BDF2 (with Lagrange interpolation), and the proposed method. For each method, solution point errors are computed with respect to an accurate reference solution. The reference solution in Fig. $4 \mathrm{a}$ is computed with regular TR-BDF2 using $h=0.25 \mathrm{~ns}$. The results for PSIM and PSCAD in Fig. 4b are computed with $h=0.63 \mu$ s. Because each full TR-BDF2 step consists of two internal steps, a double step size of $h=1.26 \mu \mathrm{s}$ is used by TR-BDF2 and the proposed method in Fig. 4c to allow a fair comparison. The boost converter circuit did not reach steady state yet and operates slightly in discontinuous conduction mode. As the duration of discontinuous conduction is sometimes smaller than the step

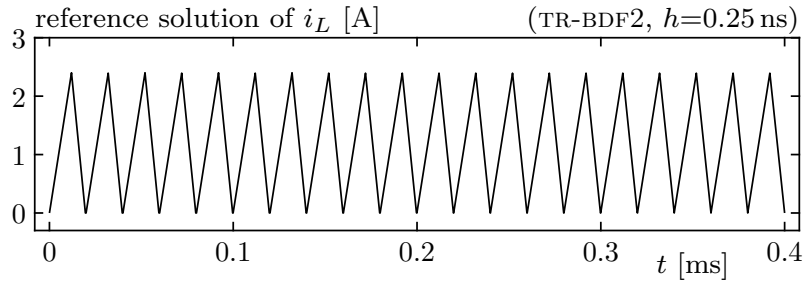

(a)

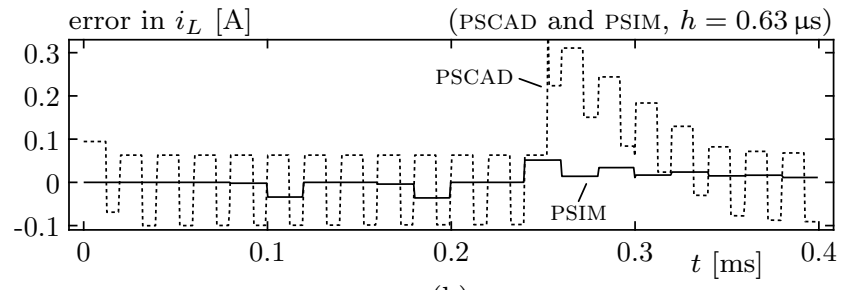

(b)

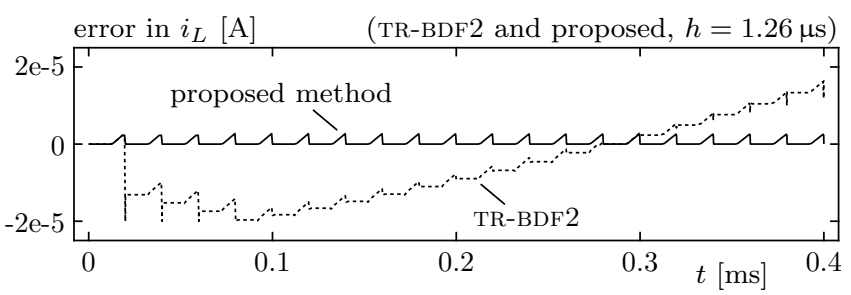

(c)

Fig. 4. Reference solution of $i_{L}$ for the circuit of Fig. 3 (a). Simulation error of PSCAD and PSIM (b). Simulation error of TR-BDF2 with regular Lagrange interpolation and the proposed method (c).

size, interpolation of the subsequent turn-on event is needed in the first step following the transition to discontinuous conduction mode. The largest error contributions of PSIM and TR-BDF2 are introduced at transitions to discontinuous conduction mode. The proposed methods does not have such errors and preserves its regular accuracy. With PSCAD, the largest error components are introduced at switch events. These errors can be attributed to inexact reinitialization. The reinitialization method of PSCAD introduces discontinuities in the dynamic variables after switching [6], [23].

In Fig. 5, the relative rms error of the solution points of $i_{L}$ and $v_{C}$ is plotted for each method for different values of the effective step size $h_{\text {eff }}$ ( $h_{\text {eff }}=h$ for PSCAD and PSIM and $h_{\text {eff }}=h / 2$ for TR-BDF2 and the proposed method). The accuracy of $i_{L}$ with PSIM varies significantly with the step size, indicating that the accuracy depends on the location of switch events within a step. The proposed method is several orders of magnitudes more accurate than PSCAD and PSIM and solves the loss of accuracy that occurs with TR-BDF2 due to inaccurate interpolation. As a consequence, it is possible to simulate this circuit with a larger step size.

\section{B. Series Load Resonant Converter in Discontinuous Conduc- tion Mode}

A second numerical experiment is conducted with the halfbridge series load resonant (SLR) converter circuit in Fig. 6, 

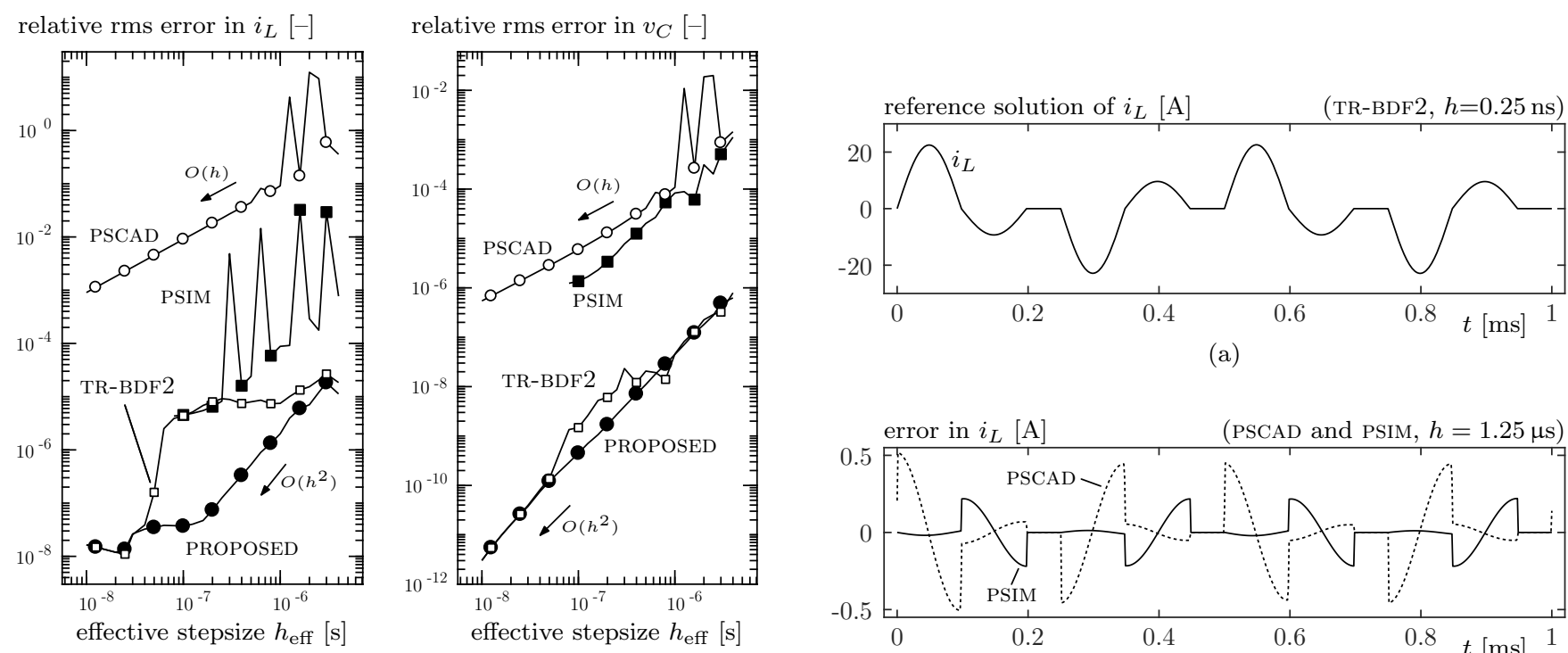

(a)

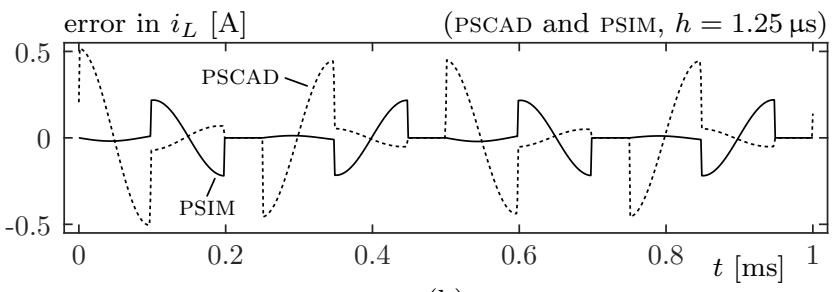

(b)

Fig. 5. Relative rms error in the simulation points as a function of the effective step size, for the variables $i_{L}$ and $v_{C}$ of the boost converter circuit in Fig. 3 . The errors are computed after simulating over $0.4 \mathrm{~ms}$ with different effective step sizes $h_{\mathrm{eff}}$, using PSCAD and PSIM with $h=h_{\mathrm{eff}}$, and TR-BDF2 and the proposed method with $h=2 h_{\text {eff }}$.

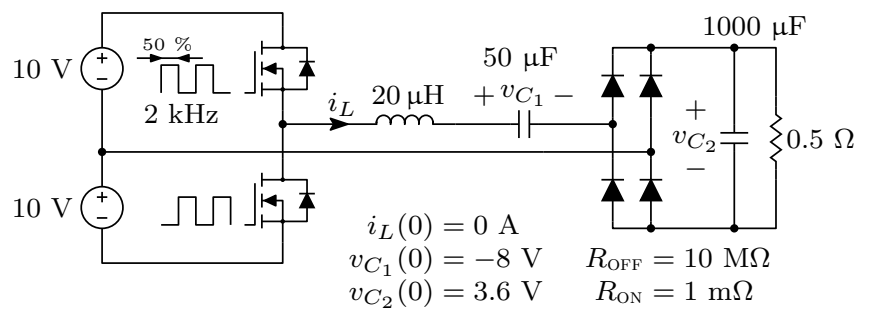

Fig. 6. Series load resonant converter test circuit with a fixed duty cycle.

also operating in discontinuous conduction mode. The circuit is simulated for $1 \mathrm{~ms}$. The reference waveform for $i_{L}$ and the errors obtained with each method are shown in Fig. 7. As this circuit involves a diode bridge, it is prone to inaccurate interpolations.

Almost immediately after the half-bridge switch event, the inductor current will force two diodes of the diode bridge to conduct. With PSCAD and TR-BDF2, the largest error is introduced at this event. With PSIM, the largest errors are introduced at commutation of the diode bridge when $i_{L}$ crosses zero. With the proposed method, there is no error visible which can be attributed to interpolation at diode bridge switch events.

In Fig. 8, the relative rms error of the solution points of $i_{L}$ and $v_{C_{2}}$ is again plotted for each method for different values of the effective step size $h_{\mathrm{eff}}$. Also for this example, the proposed method is more accurate than PSCAD and PSIM, and the regular TR-BDF2. The proposed method is the only method capable of maintaining second-order accuracy for all considered step-sizes, while the other methods suffer from a reduction to first-order accuracy. With PSIM, this order reduction occurs only for some of the considered step sizes.

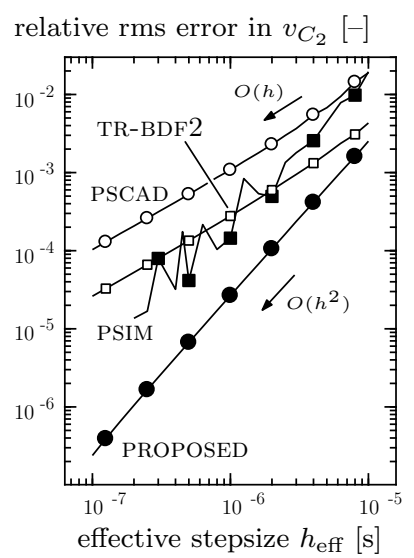

Fig. 8. Relative rms error in the simulation points as a function of the effective step size, for the variables $i_{L}$ and $V_{C_{2}}$ of the SLR converter circuit in Fig. 6. The errors are computed after simulating over $1 \mathrm{~ms}$ with different step sizes, using PSCAD and PSIM with $h=h_{\text {eff }}$ and TR-BDF2 and the proposed method with $h=2 h_{\mathrm{eff}}$. 


\section{CONCLUSION}

The proposed interpolation method for TR-BDF2 solves an accuracy problem that exists with conventional interpolation methods when interpolation is required in a step by which a very fast transient is damped. Such transients can occur in power electronic circuits if switches are modeled with small on-resistances and large off-resistances. Experimental results involving discontinuous conduction modes or diode bridges show that this problem does occur in existing tools, sometimes causing a reduction of the order of accuracy. The proposed method has three beneficial properties that improve the accuracy at switch events, often to several orders of magnitude.

First, the new method is based on second-order quadratic interpolation. Existing tools based on linear interpolation of the trapezoidal method are only first-order accurate in steps with switch events.

Second, the new method damps solution components with a very fast transient relative to the step size almost instantly, and does this in the full interpolation interval of a step. This solves the accuracy problem occurring when interpolation is required in a step by which a very fast transient is damped.

Third, the suppression of numerical oscillations is robust with interpolation. Some tools switch from the trapezoidal method to the backward Euler method to suppress such oscillations. But this method is only first-order accurate.

As it is no longer necessary to reduce the step size to compensate for the above described inaccuracies, the simulation time needed to achieve a desired level of accuracy is reduced.

\section{REFERENCES}

[1] N. Mohan, W. P. Robbins, T. M. Undeland, and R. Nilssen, "Simulation of power electronic and motion control systems-an overview," Proc. IEEE, vol. 82, no. 8, pp. 1287-1302, 1994.

[2] D. Maksimovic, A. M. Stankovic, V. J. Thottuvelil, and G. C. Verghese, "Modeling and simulation of power electronic converters," Proc. IEEE, vol. 89, no. 6, pp. 898-912, Jun. 2001.

[3] H. Jin, "Behavior-mode simulation of power electronic circuits," IEEE Trans. Power Electron., vol. 12, no. 3, pp. 443-452, 1997.

[4] J. H. Allmeling and W. P. Hammer, "PLECS - piece-wise linear electrical circuit simulation for Simulink," in Proc. Int. Conf. Power Electron. Drive Syst. Hong Kong: IEEE, 1999, pp. 355-360.

[5] H. W. Dommel and W. S. Meyer, "Computation of electromagnetic transients," Proc. IEEE, vol. 62, no. 7, pp. 983-993, Jul. 1974.

[6] N. Watson and J. Arrillaga, Power Systems Electromagnetic Transients Simulation, ser. IET power and energy series 39, A. T. Johns and D. F. Warne, Eds. London, UK: IET, 2003, vol. 39.

[7] J. Mahseredjian, V. Dinavahi, and J. A. Martinez, "Simulation tools for electromagnetic transients in power systems: Overview and challenges," IEEE Trans. Power Del., vol. 24, no. 3, pp. 1657-1669, Jul. 2009.

[8] A. M. Gole, A. Keri, C. Kwankpa, E. W. Gunther, H. W. Dommel, I. Hassan, J. R. Marti, J. A. Martinez, K. G. Fehrle, L. Tang, M. F. McGranaghan, O. B. Nayak, P. F. Ribeiro, R. Iravani, and R. Lasseter, "Guidelines for modeling power electronics in electric power engineering applications," IEEE Trans. Power Del., vol. 12, no. 1, pp. 505-514, 1997.

[9] A. M. Gole, "Electromagnetic transient simulation of power electronic equipment in power systems: challenges and solutions," 2006 IEEE Power Eng. Soc. Gen. Meet., pp. 6-11, 2006.

[10] R. J. Dirkman, "The simulation of general circuits containing ideal switches," in 1987 IEEE Power Electron. Spec. Conf., vol. 53. IEEE, Jun. 1987, pp. 185-194.
[11] D. Bedrosian and J. Vlach, "Time-domain analysis of networks with internally controlled switches," IEEE Trans. Circuits Syst. I Fundam. Theory Appl., vol. 39, no. 3, pp. 199-212, Mar. 1992.

[12] B. De Kelper, L. A. Dessaint, K. Al-Haddad, and H. Nakra, "A comprehensive approach to fixed-step simulation of switched circuits," IEEE Trans. Power Electron., vol. 17, no. 2, pp. 216-224, Mar. 2002.

[13] PLECS User Manual, Plexim GmbH, 2015, version 3.7. [Online]. Available: http://www.plexim.com/plecs

[14] SimPowerSystems User's Guide (Specialized Technology), HydroQuébec and The MathWorks, Inc, Sep. 2015, version 6.4 (Release 2015b). [Online]. Available: http://www.mathworks.com/ products/simpower/

[15] E. Hairer and G. Wanner, Solving Ordinary Differential Equations II. Springer, 1996.

[16] J. R. Marti and J. Lin, "Suppression of numerical oscillations in the EMTP," IEEE Trans. Power Syst., vol. 4, no. 2, pp. 739-747, May 1989.

[17] J. Mahseredjian and C. Dewhurst, EMTP-RV User Manual, Powersys, Jan. 2016, version 3.3.

[18] J. D. Lavers, H. Jin, and R. W. Y. Cheung, "Analysis of power electronic circuits with feedback control : a general approach," Electr. Power Appl. IEE Proc. B, vol. 137, no. 4, 1990.

[19] T. Noda, K. Takenaka, and T. Inoue, "Numerical integration by the 2-stage diagonally implicit RungeKutta method for electromagnetic transient simulations," IEEE Trans. Power Del., vol. 24, no. 1, pp. 390-399, Jan. 2009.

[20] M. E. Hosea and L. F. Shampine, "Analysis and implementation of TRBDF2,” Appl. Numer. Math., vol. 20, pp. 21-37, 1996.

[21] A. M. Gole and I. T. Fernando, "Modeling of power electronic apparatus: Additional interpolation issues," in Proc. Int. Conf. Power Syst. Transients, Seattle, WA, USA, Jun. 1997, pp. 23-28.

[22] P. Kuffel, K. Kent, and G. Irwin, "The implementation and effectiveness of linear interpolation within digital simulation," Int. J. Electr. Power Energy Syst., vol. 19, no. 4, pp. 221-227, May 1997.

[23] M. Zou, J. Mahseredjian, G. Joos, B. Delourme, and L. Gérin-Lajoie, "Interpolation and reinitialization in time-domain simulation of power electronic circuits," Electr. Power Syst. Res., vol. 76, no. 8, pp. 688-694, May 2006.

[24] L. F. Shampine and M. W. Reichelt, "The matlab ode suite," SIAM J. Sci. Comput., 1997.

[25] M. Günther and U. Feldmann, "CAD-based electric-circuit modeling in industry I. Mathematical structure and index of network equations," Surv. Math. Ind., vol. 8, pp. 97-129, 1999.

[26] K. E. Brenan, S. L. Campbell, and L. R. Petzold, Numerical Solution of Initial-Value Problems in Differential-Algebraic Equations. Siam, 1996, vol. 14.

[27] L. F. Shampine, M. W. Reichelt, and J. A. Kierzenka, "Solving index-1 DAEs in MATLAB and Simulink," SIAM Rev., vol. 41, no. 3, pp. 538-552, Jan. 1999.

[28] R. Bachmann, L. Brüll, T. Mrziglod, and U. Pallaske, "On methods for reducing the index of differential algebraic equations," Comput. Chem. Eng., vol. 14, no. 11, pp. 1271-1273, 1990.

[29] S. L. Campbell, Singular Systems of Differential Equations. Pitman, 1980, vol. 1.

[30] W. Hammer, "Dynamic modeling of line and capacitor commutated converters for HVDC power transmission," Ph.D. dissertation, ETH Zürich, 2003.

[31] K. G. Nichols, T. J. Kazmierski, M. Zwolinski, and A. D. Brown, "Overview of SPICE-like circuit simulation algorithms," IEE Proc. Circuits, Devices Syst., vol. 141, no. 4, p. 242, 1994.

[32] R. E. Bank, W. M. Coughran, W. Fichtner, E. H. Grosse, D. J. Rose, and R. K. Smith, "Transient simulation of silicon devices and circuits," IEEE Trans. Electron Devices, vol. 32, no. 4, pp. 1992-2007, 1985.

[33] E. Hairer, S. P. Nørsett, and G. Wanner, Solving Ordinary Differential Equations I. Springer, 1993.

[34] EMTDC User's Guide, Manitoba HVDC Research Centre, Sep. 2015, version 4.6. [Online]. Available: https://hvdc.ca/

[35] Simulink User's Guide, The MathWorks, Inc, Mar. 2016, version 8.7 (Release 2016a). [Online]. Available: www.mathworks.com/products/ simulink/ 